The day before slaughter, the animals were scanned and a photo was taken. After slaughter, genital tracts were collected and put into bags with individual sow identification. Examination of ovaries and uterus allowed to make a diagnosis of the status of the reproductive organs. These results were compared to the observations recorded during scanning. This gave rise to diagnoses of 9 cases of pregnancy, 19 cases of pregnancy without apparent abnormality and 13 cases of pathological abnormalities.

The accuracy of the diagnosis reached 92 p. 100 in sows without pathological abnormality or in pregnant ones. It was lower $(38.5$ p. 100$)$ in those exhibiting a pathological abnormality. Scanning showed that 3 sows out of 6 had very large cystic follicles (diameter $>25 \mathrm{~mm}$ ), and 2 out of 6 a pyometritis.

These preliminary results suggest that the diagnosis of more or less severe lesions of the genital tract will become more and more accurate as experience will be gained.

\title{
Communal rearing of the young within the social group of European wild boars
}

\author{
Isabelle DELCROIX *, I.P. SIGNORET **, R. MAUGET * \\ * Centre d'Etudes Biologiques des Animaux Sauvages (C.E.B.A.S./C.N.R.S.), \\ Villiers-en-Bois, 79360 Beauvoir-sur-Niort \\ ** I.N.R.A., Station de Physiologie de la Reproduction, \\ Nouzilly, 37380 Monnaie
}

The conditions of communal rearing of the young within the social group of European wild boars were studied in a group of six females and one male living in a wooded enclosure of 1 ha in the Chizé forest (Midwestern France).

Farrowing was recorded from early April to mid-July. Spontaneous adoptions of young occurred. They concerned three females, i.e. two which had farrowed simultaneously and a third one which had already been suckling for 21 days.

The sequence and duration of suckling were characterized. The behaviour leading to dietary independency settled parallel to suckling.

The social rearing context of the offspring was studied. The females rearing their young all together were in tight association. The different litters showed large affinities for all the mothers without any clearly selective relationships.

The absence of an early and exclusive mother-young bond seems to be in the favour of a communal care-taking of the young which in wild suids is based on the social group. 